\title{
Drift kinetic effects and local current drive induced modification of magnetic shear on sawtooth activity in EU DEMO
}

\author{
Lina Zhou ${ }^{1}$, Yueqiang Liư ${ }^{2}$, Mattia Siccinio ${ }^{3,4}$, Emiliano Fable ${ }^{4}$, Tingting $\mathrm{Wu}^{5}$, Ping Duan ${ }^{1}$, Long \\ Chen $^{1}$
}

1 College of Science, Dalian Maritime University, Dalian 116026, China

2 General Atomics, PO Box 85608, San Diego, CA 92186-5608, United States of America

3 EUROfusion Programme, Management Unit, Boltzmannstr 2, D-85748 Garching, Germany

4 Max-Planck-Institut für Plasmaphysik, Boltzmannstr. 2, 85748 Garching bei München, Germany

5 Southwestern Institute of Physics, PO Box 432, Chengdu 610041, China

E-mail: zhoulina@dlmu.edu.cn, $\underline{\text { liuv@ } @ \text { fusion.gat.com }}$

\begin{abstract}
The sawtooth activity is investigated for an EU DEMO reference plasma, including kinetic effects from both thermal particles and fusion-born alphas. Kinetic effects are studied in conjunction with modification of the magnetic shear near the $q=1$ surface ( $q$ is the safety factor) due to local current drive. Kinetic stabilization on the internal kink mode is found by both the non-perturbative MHDkinetic hybrid code MARS-K [Liu et al., Phys. Plasmas 15, 112503 (2008)] and the perturbative semi-analytic Porcelli model. The latter predicts full stabilization of the mode, when the local magnetic shear $s_{1}$ at the $q=1$ surface is less than 1 (e.g. $s_{1} \sim 0.6$ as is the case for the target EU DEMO plasma without local current drive). By increasing the local magnetic shear with local current drive up to $s_{1} \sim 2.8$, one of the Porcelli sawtooth crash criteria can be satisfied, by accessing the ion-kinetic regime. Direct MARS-K non-perturbative eigenvalue computations, on the other hand, predict less kinetic stabilization of the internal kink and more robust triggering of the sawtooth crash in EU DEMO. The general trend of the predicted results remains similar though, between the MARS-K model and the Porcelli model.
\end{abstract}

Keywords: local magnetic shear, kinetic effect, sawtooth, internal kink instability, EU DEMO

(Some figures may appear in colour only in the online journal)

\section{Introduction}

It is well known that the sawtooth activity is characterized by periodic oscillations of the core plasma 
parameters in tokamaks, such as the plasma temperature and density [1]. In a tokamak fusion reactor, small sawtooth oscillations can be beneficial, by helping prevent accumulation of helium ash and impurities with high charge number in the plasma core [2]. However, large sawtooth crashes, also often called monster sawteeth, which in turn are due to energetic particle stabilization [3, 4], can seed neoclassical tearing modes (NTMs) even at relatively low values of plasma pressure. Large NTMs can strongly degrade fusion energy confinement, and in worst case even causing plasma disruption [5, 6]. It is thus desirable to avoid monster sawteeth in reactor scale tokamak devices.

An important contributor to monster sawteeth is thought to be the fusion born $3.52 \mathrm{MeV} \alpha$ particles, which have been shown to provide a strong stabilization on the internal kink mode in ITER [7-11]. The similar situation is likely to apply to the European demonstration fusion reactor (EU DEMO) [12-14]. Therefore, understanding sawtooth behavior and its control, in the presence of alphas, represent a critical issue for EU DEMO.

The sawtooth crash is initiated by the onset of the $m=n=1$ internal kink instability, where $m$ and $n$ are the poloidal and toroidal mode numbers, respectively. Extensive research work has previously been devoted to study various physics aspects associated with the internal kink instability. For instance, it has been established that internal kink can be stabilized by reducing the plasma resistivity [15], by placing an ideal conducting wall close to the plasma [16], and by including various kinetic effects from bulk ions [7], electrons [17], fast ions [3, 18] and fusion-born $\alpha$-particles [19]. In particular, since a large amount of high energy alphas in a fusion reactor are expected to provide deep stabilization to the internal kink, creating long period, large amplitude sawteeth, techniques are required to destabilize the mode. Fortunately, it has previously been observed that sawtooth control is achievable by locally perturbing the plasma current density profile near the $q=1$ surface [20, 21]. Based on the Porcelli model model [7], simulation results for JET experiments showed that the sawtooth period is sensitive to the local change of the magnetic shear $\left(s_{1}\right)$ near the $q=1$ surface, and the internal kink mode is destabilized by increasing $s_{1}$ [22-25].

Although significant research results have so far demonstrated the capability of avoiding fastion-stabilized large sawteeth by modifying the local magnetic shear, the robustness of this technique remains an open issue. This is due to the presence of a substantial population of fusion-born $\alpha$ particles, meaning a large fraction of kinetic contribution to the perturbed potential energy $[8,10]$, in EU DEMO-like reactor scale devices with burning plasma conditions. This motivates our specific investigation into this issue, based on a reference plasma designed for EU DEMO.

In this work, full toroidal modeling of the internal kink instability is carried out for EU DEMO. Drift kinetic contributions from $\alpha$-particles, as well as that from thermal particles, are computed by the MHD-kinetic hybrid code MARS-K in a non-perturbative manner [26]. For the purpose of investigating the effect of the local magnetic shear on the sawteeth triggering, the EU DEMO reference equilibrium is also slightly (and self-consistently) modified, by adding a locally driven current perturbation (e.g. by electron cyclotron current drive) to the toroidal current density. With varying $s_{1}$, sawteeth crash conditions are assessed with the Porcelli model. We emphasize that one of the key parameters in the Porcelli model, i.e. the perturbed potential energy (with or without kinetic contributions), are evaluated by the toroidal code MARS-K, instead of adopting large aspect 
ratio analytic approximation.

An important reason for using the semi-analytic Porcelli model (but based on the MARS-K computed perturbed potential energies), for this EU DEMO study, is the inclusion of a reasonably complete set of physics in the Porcelli model, in particular the diamagnetic stabilization and the weakly unstable ion-kinetic regime, where the plasma resistive layer response plays an important role [7]. These physics are not included into the MARS-K model, which is based on the standard single fluid formulation. On the other hand, results from MARS-K direct eigenvalue computations are also reported in this work for comparative purpose. The MARS-K stability computations show generally the same trend in the kinetic stabilization of the internal kink mode, as that of the Porcelli model prediction.

This paper is organized as follows. Section 2 briefly describes the MARS-F/K computational models. Results for the EU DEMO study, including that from both MARS-K and the Porcelli model, are reported in section 3. Section 4 summarizes the work.

\section{Computational models}

The MARS-F/K codes [26, 27] are utilized to compute the growth rates and the perturbed potential energies of the internal kink mode for EU DEMO. The codes have been successfully benchmarked against other codes $[28,29]$, and have previously been applied to investigate kinetic effects on internal kink [15, 17] and external kink modes (the resistive wall modes) [30-34]. Below, we provide a brief description of the model including a self-consistent drift kinetic closure (MARS-K). The governing equations for the plasma are written for the perturbed quantities $\boldsymbol{\xi}, \boldsymbol{v}, \boldsymbol{b}, \boldsymbol{j}, \boldsymbol{p}$, representing the plasma displacement, perturbed velocity, magnetic field, current density and pressure, respectively

$$
\begin{gathered}
(\tilde{\gamma}+i n \Omega) \boldsymbol{\xi}=\boldsymbol{v}+(\boldsymbol{\xi} \cdot \nabla \Omega) R^{2} \nabla \boldsymbol{\phi} \\
\rho(\tilde{\gamma}+i n \Omega) \boldsymbol{v}=-\nabla \cdot \boldsymbol{p}+\boldsymbol{j} \times \boldsymbol{B}+\boldsymbol{J} \times \boldsymbol{b} \\
-\rho\left[2 \Omega \hat{\boldsymbol{Z}} \times \boldsymbol{v}+(\boldsymbol{v} \cdot \nabla \Omega) R^{2} \nabla \boldsymbol{\phi}\right]-R \Omega^{2} \nabla \cdot(\rho \boldsymbol{\xi}) \hat{\boldsymbol{R}} \\
(\tilde{\gamma}+i n \Omega) \boldsymbol{b}=\nabla \times(\boldsymbol{v} \times \boldsymbol{B})+(\boldsymbol{b} \cdot \nabla \Omega) R^{2} \nabla \boldsymbol{\phi}-\nabla \times(\eta \boldsymbol{j}) \\
(\tilde{\gamma}+i n \Omega) p=-\boldsymbol{v} \cdot \nabla P \\
j=\nabla \times \boldsymbol{b} \\
\boldsymbol{p}=p \boldsymbol{I}+p_{\|} \hat{\boldsymbol{b}} \hat{\boldsymbol{b}}+p_{\perp}(\boldsymbol{I}-\hat{\boldsymbol{b}} \hat{\boldsymbol{b}}) \\
p_{\mathrm{P}}=\sum_{j} M_{j} v_{\mathrm{P}}^{2} f_{L}^{1} d \Gamma, p_{\perp}=\sum_{j} \frac{1}{2} M_{j} v_{\perp}^{2} f_{L}^{1} d \Gamma
\end{gathered}
$$

where $\rho, \boldsymbol{B}$ and $\boldsymbol{J}$ are the equilibrium plasma density, magnetic field and current density, respectively. $P$ is the total equilibrium pressure. The linear stability problem is formulated as an eigenvalue 
problem with $\tilde{\gamma}$ being the eigenvalue, and its real and imaginary parts refer to the growth rate and the mode frequency, respectively. In the presence of the plasma equilibrium toroidal rotation, the eigenvalue is corrected by a Doppler shift frequency in $\Omega$, with $\Omega$ bring the angular frequency of the flow along the geometric toroidal angle $\phi$ of the torus. $\hat{Z}$ and $\hat{R}$ are the unit vectors in the vertical and radial directions, respectively, in the poloidal plane, and $R$ is the plasma major radius. The plasma resistivity is denoted by $\eta$. The Spitzer model is used for the plasma resistivity, which gives the on-axis Lundquist number of $\sim 10^{9}$ for this EU DEMO plasma.

The drift kinetic effects self-consistently enter the MHD equations via the perturbed kinetic pressure tensor $\boldsymbol{p}$ in Eq. (2). As shown in Eq. (6), the perturbed pressure tensor $\boldsymbol{p}$ consists of a scalar component $p$, and anisotropic tensor components describing the perturbed drift kinetic pressure (the non-adiabatic contributions) parallel $\left(p_{\mathrm{P}}\right)$ and perpendicular $\left(p_{\perp}\right)$ to the equilibrium field line. The symbol $\boldsymbol{I}$ denotes the unit tensor here, and $\hat{\boldsymbol{b}}=\boldsymbol{B} /|B|$. The kinetic pressure perturbations are calculated from Eq. (7), where $\Gamma$ signifies the velocity space of the particles, and $j$ represents the particle species, including the thermal ions and electrons as well as the fusion born $\alpha$-particles in this study. $M_{\mathrm{j}}$ is the corresponding particle mass. $v_{\mathrm{P}}$ and $v_{\perp}$ denote the parallel and perpendicular velocities of the particle guiding center drift motion, respectively. We assume a Maxwellian equilibrium distribution function for thermal particles, and an isotropic slowing-down equilibrium distribution function for $\alpha$-particles. $f_{L}^{1}$ is the non-adiabatic perturbed distribution function, which contains a key factor, i.e., the mode-particle resonance operator

$$
\lambda_{m l}=\frac{n\left[\omega_{*_{N}}+\left(\hat{\varepsilon}_{k}-\frac{3}{2}\right) \omega_{*_{T}}+\omega_{E}\right]-\infty 0}{n \omega_{d}+[\alpha(m+n q)+l] \omega_{b}-i v_{e f f}-\varnothing c}
$$

where $\omega_{*_{N}}$ and $\omega_{*_{T}}$ are the diamagnetic drift frequencies associated with the plasma density and temperature gradients, respectively. $\omega_{E}$ is the $\boldsymbol{E} \times \boldsymbol{B}$ drift frequency due to the equilibrium electrostatic potential. $\omega_{d}$ is the bounce-orbit-averaged toroidal precession drift frequency of particles, including the $\omega_{E}$ drift. $\omega_{b}$ is the particle bounce/transit frequency. $\hat{\varepsilon}_{k}$ is the particle kinetic energy normalized by the temperature. $\not \sigma=i q /$ is the mode frequency. $l$ is the Fourier harmonic indices over the particle bounce orbit. $\alpha=1$ for passing particles, and $\alpha=0$ for trapped particles. $v_{\text {eff }}$ is the effective particle collision frequency.

For the EU DEMO equilibrium, plasma flow is expected to be relatively slow. Therefore, kinetic contributions from passing particles are neglected in view of weak resonance between the internal kink mode and the fast transit motion of thermal ions. The transit motion of thermal electrons or $\alpha$-particles is even faster, inducing even weaker effect and is thus also neglected. In addition, the kinetic contribution due to (fast) bounce motion of trapped thermal electrons is also ignored.

\section{Numerical results}

The EU DEMO equilibrium is briefly introduced in sub-section 3.1. Sub-section 3.2 studies the 
effect of edge equilibrium current on the equilibrium profiles in particular that of the safety factor. In the following sub-sections 3.3 and 3.4, an equilibrium with vanishing edge current is adopted for the internal kink study, in order to avoid interference from the edge-current driven peeling component (with dominant harmonic $m / n=5 / 1$ ) which is not of interest in this work. The effect of conducting wall on the internal kink is weak. A close-fitting ideal wall is hence assumed in most of our investigations. Sub-section 3.3 investigates the effect of local magnetic shear on the fluid internal kink. Similar effect is studied in sub-section 3.4 with inclusion of drift kinetic effects.

\subsection{Plasma equilibrium for an EU DEMO scenario}

A reference plasma equilibrium from the EU DEMO design [35] is adopted for this study. The key equilibrium parameters are: the plasma major radius is $R_{0}=9 \mathrm{~m}$ and the aspect ratio $A=R_{0} / a=3.1$ ( $a$ being the plasma minor radius), the on-axis vacuum toroidal field $B_{0}=5.85 \mathrm{~T}$, the safety factor of the magnetic axis $q_{0}=0.46$. Figure 1 shows radial profiles of equilibrium quantities. The pressure (Fig. 1(a)) is normalized by $B_{0}^{2} / \mu_{0}$, with $\mu_{0}$ being the vacuum magnetic permeability. The plasma density (Fig. 1(a)) is normalized to unity at the magnetic axis. The fusion born alpha particles (Fig. 1(b)) are assumed to have isotropic equilibrium distribution in particle pitch angle and slowingdown in particle energy $[8,31]$. Note that the density and pressure profiles of alphas are normalized by the corresponding thermal electron density and total thermal pressure, respectively. For this DEMO design, $\alpha$ particles contribute about $45 \%$ fraction of the thermal pressure in the plasma core to the total equilibrium pressure, with only about $2.5 \%$ of density fraction. It is important to point that, in our following studies on the internal kink, the total equilibrium pressure is kept constant. In other words, $P_{\mathrm{eq}}=P_{\mathrm{th}}+P_{\alpha}$ in the presence of alphas; and in the absence of alphas, the portion of equilibrium pressure associated with $P_{\alpha}$ is effectively replaced by the thermal contribution. Thus ensures that the "fluid" drive is the same for the mode studied in this work.

The equilibrium toroidal current density is slightly modified from the original DEMO design. Figures 1(c-d) compare radial profiles of the surface averaged toroidal current density and the corresponding safety factor, between the original (solid blue, $\mathrm{J}_{1}$ ) and the modified (dashed red, $\mathrm{J}_{2}$ ) versions. The latter is obtained by slightly modifying the $\mathrm{J}_{1}$ profile near the plasma edge, to make vanishing equilibrium current at the plasma surface. As will be shown later on, this slight modification does not affect much the internal kink stability analysis, but helps to avoid the excitation of an edge-localized kink instability that is undesirable in our eigenvalue computations. The radial location of the $q=1$ surface, $r_{1}=0.38 a$ as indicated by the dashed vertical line in Fig. 1(d), is farther away from the plasma edge. The influence of this slight edge current density change to the internal kink instability is thus minimal. We emphasize that the Grad-Shafranov equation is resolved for the modified current profile, so that a self-consistent (modified) equilibrium is generated for further stability analysis. 


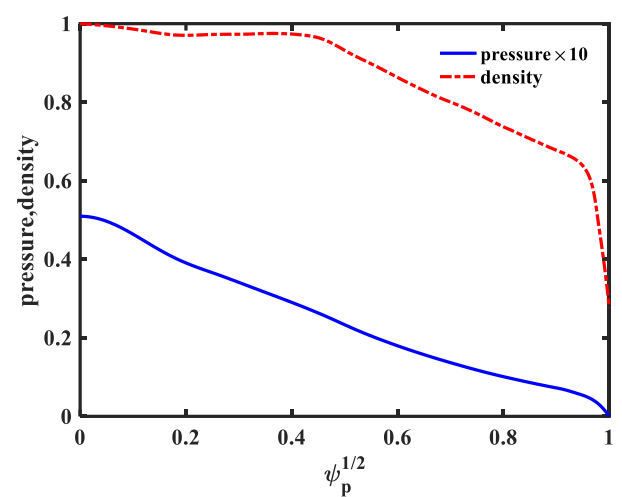

(a)

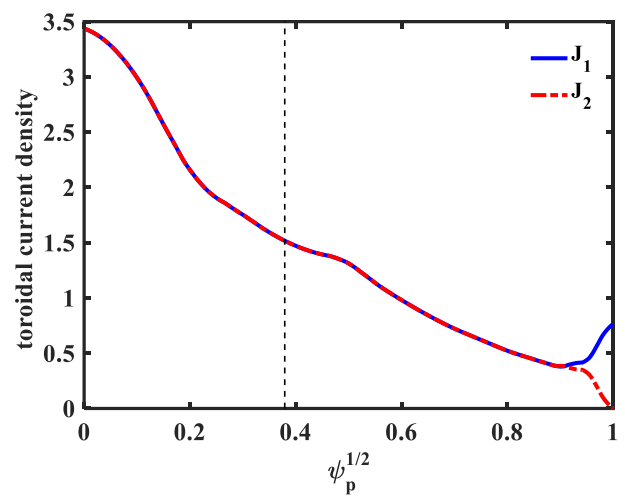

(c)

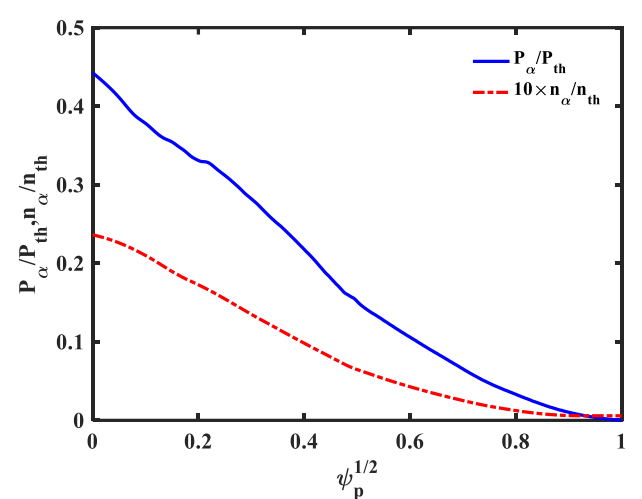

(b)

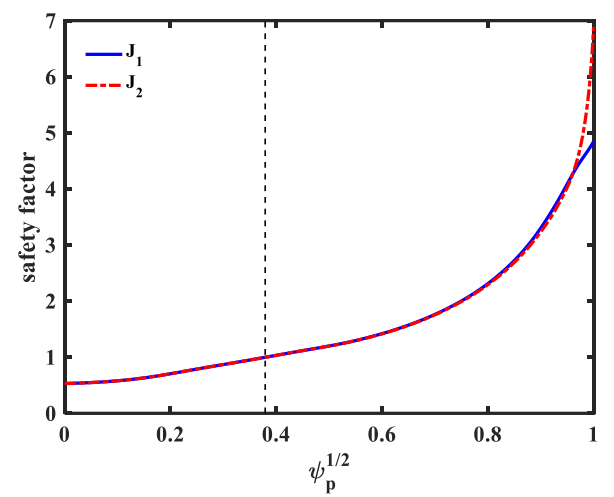

(d)

Figure 1. The equilibrium radial profiles for an EU DEMO reference plasma, for (a) the plasma pressure normalized by $B_{0}^{2} / \mu_{0}$ and the density normalized to unity at the magnetic axis, (b) the density and pressure fractions due to fusion born $\alpha$-particles with respect to the electron density and the thermal pressure, respectively, (c) the two choices of the surface averaged toroidal current density normalized by $R_{0} B_{0} / \mu_{0}$, and (d) the two safety factors $q$, corresponding to the two current density profiles from (c). $\psi_{\mathrm{p}}$ is the equilibrium poloidal magnetic flux, normalized to $\psi_{\mathrm{p}}=0$ at the magnetic axis and $\psi_{\mathrm{p}}=1$ at the plasma boundary. The vertical dashed lines in plots (c-d) indicate the radial location of the $q=1$ surface.

\subsection{Effects of ideal wall radial location and edge toroidal current}

It is well known that both the ideal internal [16] and external [35] kink modes can be stabilized by the presence of an ideally conducting wall. A close-fitting conducting wall surounding the plasma can stabilize the ideal internal kink, even when the plasma equilibrium pressure is above the Bussac limit [15]. For the EU DEMO plasma considered here, the $m / n=1 / 1$ ideal internal kink is indeed unstable at vanishing equilibrium flow, as computed by MARS-F. As mentioned earlier, in the presence of finite edge current (model $\mathrm{J}_{1}$ in Fig. 1(c) for the original equilibrium), another unstable mode also appears by moving the ideal wall, with radial location $b$, away from the plasma surface.

Figure 2(a) reports both unstable roots for this equilibrium. One is the conventional $m / n=1 / 1$ ideal internal kink ( "J $\mathrm{J}_{1}$-root 1 "). This root is subject to relatively weak stabilization by the ideal wall. 
In fact, the root is not sensitive to edge current density profile either - nearly the same root (marked as " $\mathrm{J}_{2}$ " in Fig. 2(a)) is recovered by considering the modified equilibrium with vanishing edge current. The second root ( " $\mathrm{J}_{1}$-root $\mathrm{r}_{2}$ ") for the original equilibrium, however, is sensitive to the ideal wall location. This root is stabilized by an ideal wall located at the plasma surface, but becomes strongly unstable when the wall is moved away from the plasma. The second root becomes dominant at $b>1.15 a$. No such a root is found for the modified equilibrium with vanishing edge current density, indicating that the second root is driven by the finite equilibrium current near the plasma edge for the original equilibrium.

Analysis of the mode spectra, compared in Fig. 2(b) for the amplitude of the poloidal Fourier harmonics of the radial displacement, shows two large harmonics for the second root (" $\mathrm{J}_{1}, \mathrm{~b}=2 \mathrm{a}$, root2"), i.e. $m / n=1 / 1$ and $m / n=5 / 1$. All the other roots meanwhile only have one dominant harmonic $m / n=1 / 1$ which is the conventional internal kink component.

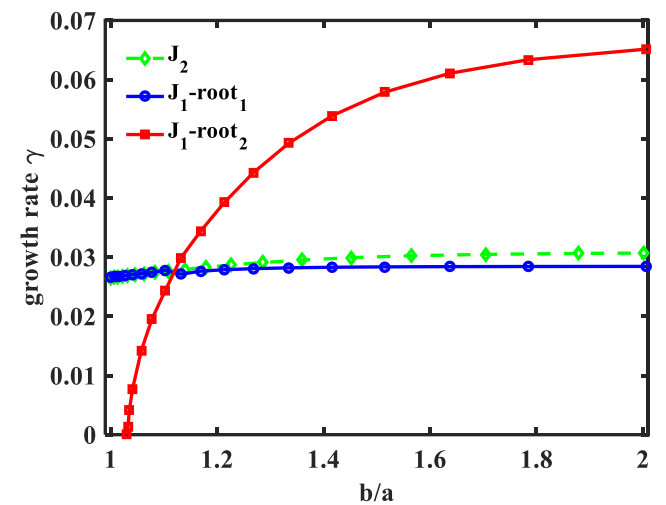

(a)

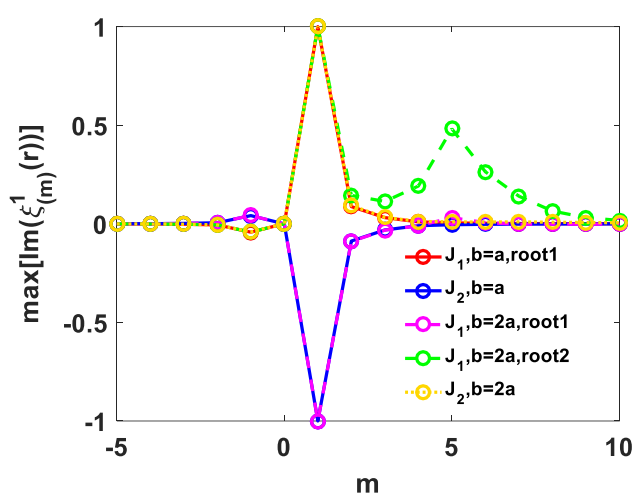

(b)

Figure 2. The MARS-F computed (a) growth rate $\gamma$ of the instabilities, normalized by the on-axis Alfven frequency $\omega_{A}=B_{0} /\left[R_{0}\left(\mu_{0} \rho_{0}\right)\right]$, with varying radial location $b / a$ of the ideal conducting wall, and (b) maximal amplitude (along the plasma minor radius) of the poloidal Fourier harmonics of the $n=1$ radial displacement $\xi^{1}$, normalized to unity for the $m / n=1 / 1$ harmonic. The legends $\mathrm{J}_{1}$ and $\mathrm{J}_{2}$ indicate the corresponding toroidal current density profiles shown in Fig. 1(c).

The $m / n=5 / 1$ component for the second unstable root is localized near the plasma edge as shown in Fig. 3(b), representing a peeling instability. In other words, the second root is a compound mode consisting of predominantly both $m / n=1 / 1$ internal kink and $m / n=5 / 1$ edge peeling components. For comparison, the eigenmode structure (the plasma radial displacement) is also plotted for the first root (Fig. 3(a)) of the original equilibrium, showing a typical internal kink. With the ultimate goal of investigating the $m / n=1 / 1$ internal kink instability in full toroidal geometry for EU DEMO in this study, we shall assume a close-fitting ideal wall $(b=a)$ in what follows, in order to suppress any peeling component. Furthermore, to ensure that no such component is triggered by other physics effects (e.g. the drift kinetic effects considered in this work), we shall adopt the modified equilibrium with vanishing current density at the plasma edge (model $\mathrm{J}_{2}$ ). The eigenstructure of the internal kink for the modified equilibrium is very similar to that shown in Fig. 3(a). 


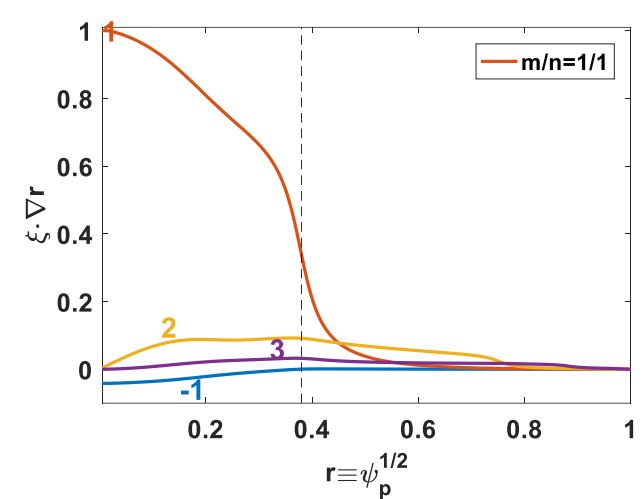

(a)

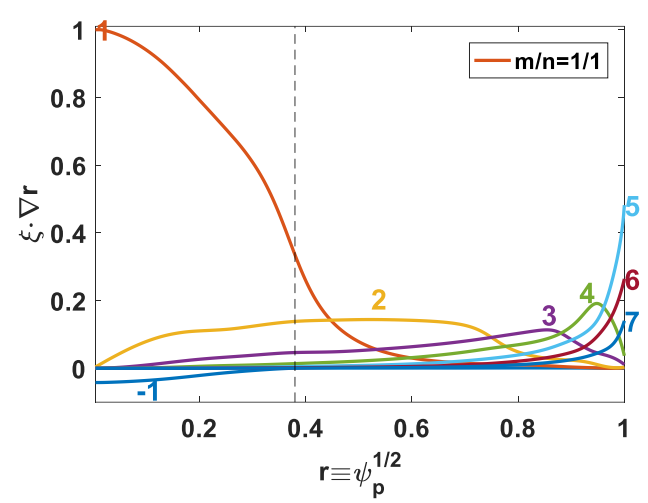

(b)

Figure 3. Comparison of the eigenmode structure, in terms of the poloidal Fourier harmonics of the radial plasma displacement, for (a) the $n=1$ ideal internal kink computed with vanishing equilibrium current density at the plasma boundary (model $\mathrm{J}_{2}$ from Fig. 1(c)), and (b) the edge localized $n=1$ ideal peeling mode driven by the edge current (model $\mathrm{J}_{1}$ from Fig. 1(c)) mixed with the internal kink component. An ideal conducting wall is placed far away from the plasma surface, radius, at $b / a=2$. The dashed vertical lines indicate the radial location of the $q=1$ surface.

\subsection{Effect of local magnetic shear at $q=1$ surface within fluid model}

It has been well established, both in theory and experiments, that the local magnetic shear near the $q=1$ surface plays a significant role in the internal kink stability, thus strongly impacting the sawtooth oscillation behavior [7, 20]. We carry out a systematic investigation of this effect here for the EU DEMO plasma. The study is based on the fluid model (MARS-F). The results with drift kinetic effects will be reported in the following sub-section.

In experiments [20,21], internal kink has been found to be destabilized by the localized current drive near the $q=1$ surface, by several methods including the electron cyclotron current drive and the ion cyclotron current drive. We model the current drive by introducing an equilibrium current perturbation with Gaussian function

$$
\delta J_{\phi}=C_{A} \cdot \exp \left[-\frac{\left(r-r_{1}\right)^{2}}{2 C_{W}{ }^{2}}\right]
$$

where $r_{1}=0.38 a$ is fixed at the radial location of the $q=1$ surface for the equilibrium labeled " $\mathrm{J}_{2}$ " as shown in Fig. 1(d). Note that the Gaussian perturbed current produced by electron cyclotron current drive for EU DEMO has been shown in the ref. [36]. With the perturbed current, new equilibria are self-consistently computed using the fixed boundary equilibrium solver CHEASE [37], with slight change to the total plasma current while at fixed toroidal magnetic field.

By tuning parameters $C_{A}$ and $C_{W}$, a series of new profiles for the safety factor and the magnetic shear are obtained as shown Fig. 4. We find that, by fixing the product of $C_{A}$ and $C_{W}$ to a constant as well as fixing $r_{1}=0.38 a$, the radial location of the $q=1$ surface for all the new equilibria stays essentially unchanged. This is important since the radial location of the $q=1$ surface has large influence on the internal kink and fishbone stability [8]. Moreover, the total toroidal plasma current 
(and hence the edge safety factor) also stays nearly the same as shown in Fig. 4(a). Figure 4 shows four representative cases, with the black curves $\left(s_{1}=0.6\right)$ corresponding to the profiles without application of the local current perturbation, Eq. (9). The others three cases $\left(s_{1}=1.5,2.5\right.$ and 3.5) show progressive increase of the local magnetic shear.

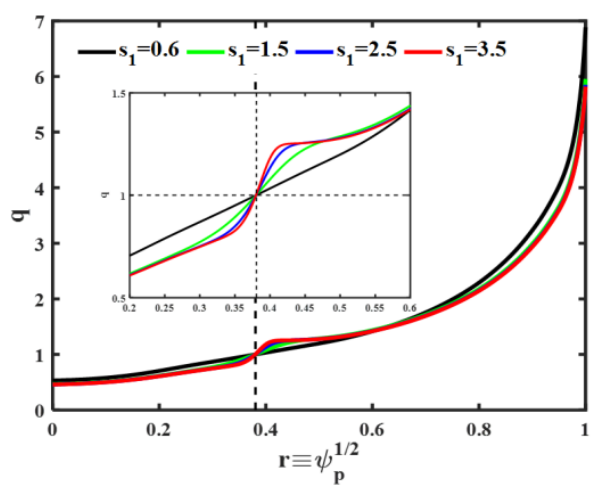

(a)

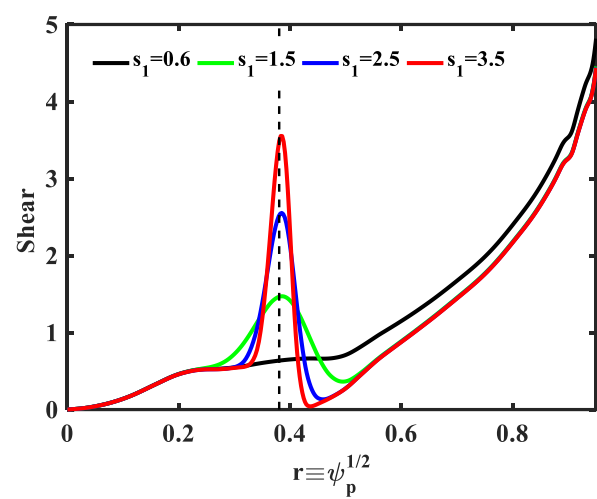

(b)

Figure 4. (a) A series of safety factor $q$-profiles with local variation of the magnetic shear $s_{1}$ at the $q=1$ surface, while fixing the radial location of the $q=1$ surface at $r_{1}=0.38$. (b) The corresponding radial profiles of the magnetic shear. The dashed vertical lines indicate the radial location of the $q=1$ surface.

Next, we study the influence of the local magnetic shear on the internal kink growth rate. Since the radial location of the ideal wall has minor effect on the mode stability for this EU DEMO plasma (Fig. 2(a)), we shall fix the ideal wall position at the plasma boundary, i.e. $b=a$. We also remark that the effect of the local magnetic shear on internal kink is qualitatively well understood in Porcelli theory. What we report here are quantitative results for a specific plasma from EU DEMO design. In addition, we shall consider the effect of the plasma resistivity as well, as shown in Fig. 5(a). Note that only the mode growth rate is shown here. The mode frequency remains vanishing without plasma equilibrium flow (which we assume) nor drift kinetic resonance effects. The key observations from Fig. 5(a) are the following. (i) At low plasma resistivity (including the ideal plasma limit), the local magnetic shear generally stabilizes the internal kink. A slight destabilization, however, occurs at large magnetic shear. (ii) The dependence is different for a highly resistive plasma (which is of course not DEMO-relevant but nevertheless of theoretical interest), in which case the local magnetic shear always destabilizes the mode. (iii) At fixed magnetic shear, the plasma resistivity destabilizes the internal kink - a result which is well known (see e.g. Ref. 14).

Our further investigation of the internal kink stability and sawtooth behavior, based on the Porcelli model [7], requires computation of the perturbed MHD potential energy $\delta W_{\text {mhd. }}$. The latter is plotted against variation of the local magnetic shear in Fig. 5(b), while fixing the (normalized) plasma resistivity at $\eta=10^{-9}$, which is the value estimated for the EU DEMO plasma considered in this study. We remark that the same order of magnitude for the plasma resistivity was also assumed in a sawtooth study for ITER [7]. We also remark that the real part of $\delta W_{\text {mhd }}$ is normalized 
by the product of the plasma inertia $\delta I$ (to be defined later) and the mode growth rate $\gamma$, with the latter being normalized by the on-axis toroidal Alfven frequency. The imaginary part of $\delta W_{\text {mhd }}$ vanishes within the fluid approximation. The normalized perturbed MHD potential energy scales linearly with the local magnetic shear (at least at reasonably low shear values), as shown by Fig. $5(b)$.

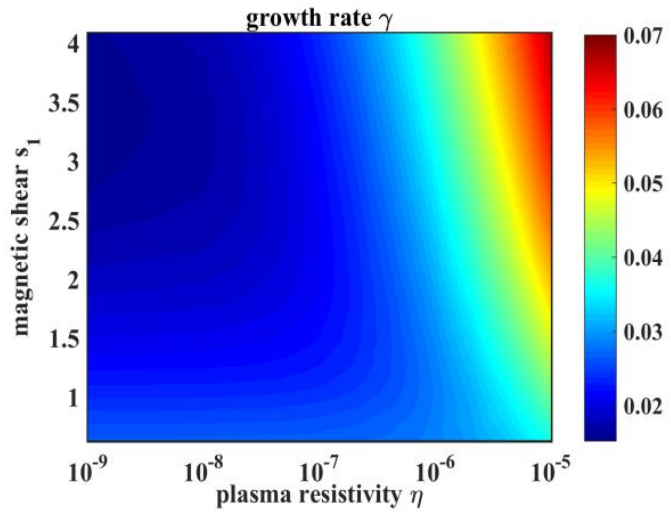

(a)

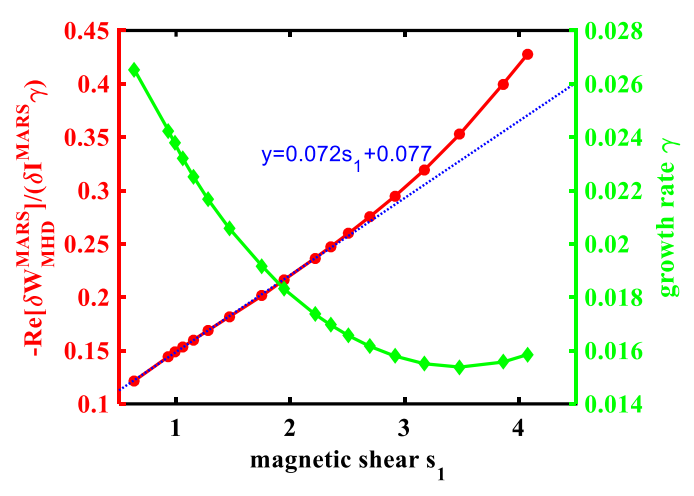

(b)

Figure 5. (a) The MARS-F computed growth rate of the $n=1$ resistive internal kink instability, normalized by the on-axis Alfven frequency, with varying plasma resistivity $\eta$ and magnetic shear $s_{1}$ at the $q=1$ surface. The resistivity $\eta$ is normalized by , such that $1 / \eta$ corresponds to the Lundquist number. (b) The computed MHD potential energy $\delta W_{M H D}^{M A R S}$, normalized by the product of the plasma inertia $\delta I$ associated with the radial displacement and the growth rate $\gamma$ (also shown by the second vertical axis on the right) normalized by the on-axis Alfven frequency, versus the magnetic shear $s_{1}$ at fixed $\eta=1 \times 10^{-9}$. Presented in (b) is also an analytic fitting of the normalized potential energy. An ideal wall is placed at the plasma boundary: $b / a=1$.

In order to better cast the MARS-F computational results into the Porcelli model, we notice that the mode is strongly unstable, with the normalized growth rate $\gamma$ being of order $10^{-2}$ despite the stabilizing effect from the enhanced magnetic shear at the $q=1$ surface. This indicates that the internal kink instability in this EU DEMO plasma is mainly determined by the perturbed potential energy, and less by the resistive layer physics around the $q=1$ surface. The layer physics effects become unimportant when the following condition is satisfied [7]

$$
-\delta \hat{W}>-\delta \hat{W}_{c}=\max \left\{\hat{\rho}, \omega_{*}^{t h i} \tau_{A} / 2\right\}
$$

where $\hat{\rho}=\rho_{i} / r_{1}$, with $\rho_{i}$ being the thermal ion Larmor radius. $\omega_{*}^{\text {thi }}$ indicates the thermal ion diamagnetic frequency, and $\tau_{A}$ the Alfven time. The values of the relevant parameters are listed in Tab. 1. $\delta \hat{W}$ signifies the normalized potential energy

$$
\delta \hat{W} \propto \frac{\delta W}{\delta I \cdot s_{1}}
$$

where $\delta W$ represents the perturbed potential energy and $\delta I$ refers to the inertial energy associated 
with the radial displacement of the plasma alone. These two energy components are computed by MARS-F and denoted as $\delta W_{M H D}^{M A R S}$ and $\delta I^{M A R S}$, respectively, in Fig. 5(b). According to the Porcelli model, the mode growth rate, normalized by the Alfven time, reduces to $\gamma \approx-\delta \hat{W}$ when the layer physics becomes negligible [7]. It is clear that $-\delta \hat{W}$ in the Porcelli model is positive and decreases with increasing $s_{1}$ (Eq. (11)), reflecting the stabilizing effect of the local magnetic shear on the internal kink mode.

On the other hand, Fig. 5(b) shows a roughly linear analytic fitting for the MARS-F computed MHD energy perturbation as a function of the local magnetic shear,

$$
-\frac{\operatorname{Re}\left[\delta W_{M H D}^{M A R S}\right]}{\operatorname{Re}\left[\delta I^{M A R S}\right] \cdot \gamma} \approx 0.072 s_{1}+0.077,
$$

allowing us to introduce the following normalization

$$
-\delta \hat{W} \cong-\frac{\operatorname{Re}[\delta W]}{\operatorname{Re}[\delta I] \cdot\left(0.072 s_{1}+0.077\right)},
$$

in order to match that of the Porcelli model. In other words, both Eqs. (11) and (13) ensure $\gamma=-\delta \hat{W}$. We emphasize that it is important to introduce the normalization in Eq. (13), in order to allow us to apply the MARS-F computed $\delta \hat{W}$ to the Porcelli sawtooth model later on, when kinetic effects from EPs are included. We also note the slight difference in the form between Eqs. (11) and (13). In particular, a shifting constant of 0.077 is needed in the shear factor, in order to fit well the MARS-F computed mode growth rate (Fig. 5(b)), as compared to that of the simple analytic model in Eq. (11). Finally, we mention that these fitting coefficients around the magnetic shear only weakly depend on the plasma resistivity assumed in the MARS-F computations.

Table 1. Relevant parameters of layer physics effect criterion in EU DEMO

\begin{tabular}{lll}
\hline \hline thermal ion Larmor radius & $\rho_{i}=v_{\text {thi }} / \Omega_{c i}$ & $0.5 \mathrm{~cm}$ \\
radius of the $q=1$ surface & $r_{1}$ & $1.1 \mathrm{~m}$ \\
thermal ion diamagnetic frequency & $\omega_{*}^{\text {thi }}=T / e B r_{P} \rho_{1}$ & $3.2 \times 10^{3} \mathrm{~s}^{-1}$ \\
Alfven time & $\tau_{A}=\sqrt{3} R / v_{A}$ & $1.9 \mu \mathrm{s}$ \\
\hline \hline
\end{tabular}

\subsection{Effect of local magnetic shear at $q=1$ surface with drift kinetic effects}

In what follows, we investigate the effect of local magnetic shear on the internal kink instability, in the presence of drift kinetic effects. Considered are the kinetic contributions from the precessional drift resonance due to trapped thermal ions and electrons $\left(t_{\mathrm{P}}\right)$, the bounce resonance due to trapped thermal ions $\left(t_{\mathrm{B}}\right)$ and the precessional drift resonance due to trapped fusion born alpha particles $\left(\alpha_{\mathrm{P}}\right)$ in EU DEMO. As mentioned in section 2, we ignore the kinetic contributions from bounce motion of trapped thermal electrons, as well as transit motions of passing thermal particles and fusion alphas, due to their weak resonances with the internal kink. Finite orbit width (FOW) corrections of alpha particles are neglected as a model simplification. This effect may be important near the $q=1$ surface, 
where the internal kink experiences large variation in the mode structure. On the other hand, toroidal computations (Fig. 3) reveal that the mode structure does not sharply vary near the $q=1$ surface, as has usually been assumed in theoretical models.

The kinetic contributions to the perturbed potential energy, associated with the internal kink, are computed based on the computed mode eigenfunction by the non-perturbative MHD-kinetic hybrid code MARS-K. We emphasize that the non-perturbative nature of the MARS-K formulation allows self-consistent modification of the mode eigenfunction by the drift kinetic effects. Moreover, MARS-K also directly produce the mode eigenvalue, which will be compared with that obtained from the Porcelli model. We start by reporting results for the reference EU DEMO plasma without local modification of the magnetic shear.

\subsubsection{Internal kink stability and sawtooth crash for reference plasma}

Figure 6(a) compares the MARS-K computed eigenvalues of the internal kink for the EU DEMO equilibrium without changing the local magnetic shear, with various combinations of including drift kinetic effects. The growth rate of the internal kink, shown along the vertical axis, is reduced by the drift kinetic effect from each individual particle species and type of resonance. The largest effect comes from the precessional drifts of trapped thermal particles, which results in a substantial reduction (about a factor of two) of the mode growth rate, albeit no full stabilization of the mode is achieved. The (partial) stabilization is enhanced by the combination of the precessional drift resonance due to trapped thermal particles and the bounce resonance due to trapped thermal ions, reducing the fluid growth rate to less than a third. On the other hand, the presence of high energy alpha particles slightly weakens the stabilizing effect of thermal particles, indicating a cancellation effect of the precessional drift resonances between thermal and energetic particles. We mention that similar cancellation effect has previously be found, albeit for the external kink mode (the resistive wall mode) [38, 39]. Finally, we note that drift kinetic resonances also produce finite mode frequency (in the absence of the plasma equilibrium flow), as shown by the horizontal axis in Fig. 6(a). The precession drifts from fusion born trapped alpha particles play a dominant role here.

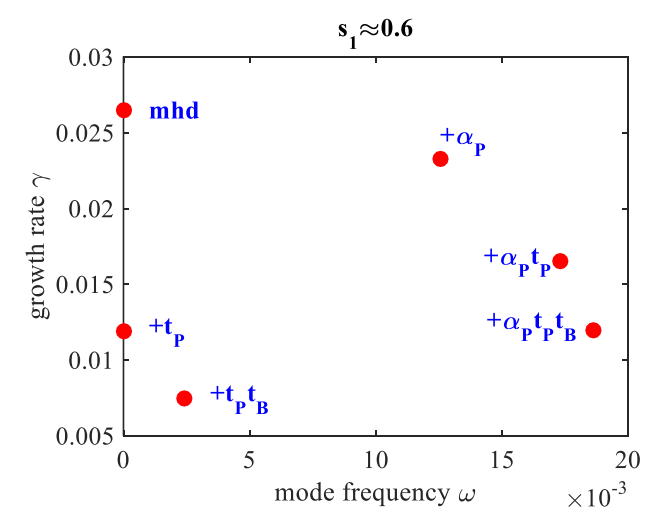

(a)

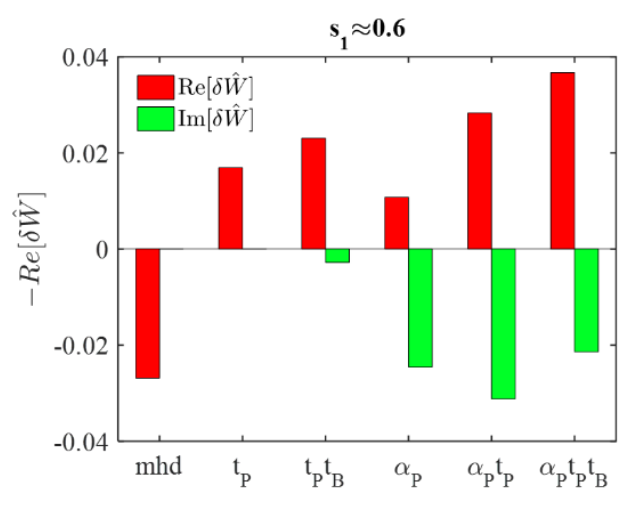

(b)

Figure 6. (a) The MARS-K computed growth rate $\gamma$ (vertical axis) and mode frequency $\omega$ (horizontal axis) for the $n=1$ internal kink instability, with inclusion of various drift kinetic effects associated with toroidal precession of trapped thermal particles $\left(t_{\mathrm{P}}\right)$ or trapped fusion born alpha 
particles $\left(\alpha_{\mathrm{P}}\right)$, and with bounce motion of trapped thermal ions $\left(t_{\mathrm{B}}\right)$. The symbol '+ 'indicates inclusion of the MHD contribution. (b) The real and imaginary parts of the normalized potential energies, associated with the MHD and drift kinetic contributions. Other parameters are $\eta=1 \times 10^{9}$, $b / a=1$.

Based on the MARS-K computed mode eigenfunction (i.e. that self-consistently modified by the kinetic effects), various components of the perturbed potential energy are also evaluated and plotted in Fig. 6(b), including the MHD contribution and the kinetic contributions from thermal particles as well as from fusion-born alphas. These energy components help to analyze the sawtooth crash based on the Porcelli model [7]. The latter predicts triggering of sawtooth crashes when one of the following conditions is satisfied:

$$
\begin{gathered}
-\delta \hat{W}_{\text {core }}>c_{h} \omega_{D \alpha} \tau_{A} \\
-\delta \hat{W}>0.5 \omega_{*}^{\text {thi }} \tau_{A} \\
-c_{\rho} \hat{\rho}<-\delta \hat{W}<0.5 \omega_{*}^{\text {thi }} \tau_{A} \text { and } \omega_{*}^{\text {thi }}<c_{*} \gamma_{\rho}
\end{gathered}
$$

where $c_{h}, c_{\rho}$ and $c_{\star}$ are the numerical factors depending on the plasma equilibrium. We choose these factors to be the same as in an ITER study [7], i.e., $c_{\mathrm{h}}=0.4, c_{\rho}=1$ and $c_{*}=1 . \omega_{D \alpha}=E / 4 e B R \bar{\rho}_{1}$ is the precessional drift frequency of alphas at the particle birth energy $E_{\alpha} \cdot \gamma_{\rho}$ is the characteristic growth rate of the internal kink instability in the ion-kinetic regime, characterized by the condition

$$
d_{e}<\delta_{\eta}<\rho_{i}
$$

where $d_{e}$ is the electron inertial skin depth and $\delta_{\eta}=s_{1}^{-1 / 3} \eta^{1 / 3} \rho_{1}$ is the resistive layer width. For the EU DEMO-like plasmas, $d_{e}$ is about one order of magnitude smaller than the thermal ion Larmor radius $\rho_{i}$, which defines the range of values for $\delta_{\eta}$. It turns out that the inequality (17) is satisfied with $\eta=1 \times 10^{-9}$ and $s_{1} \in[0.5,4.0]$, which is the parameter spaces covered by our study for the EU DEMO plasma.

The quantity $\delta \hat{W}_{\text {core }}$ from Eq. (14) represents the normalized the core plasma potential energy, composed by the perturbed fluid potential energy and the Kruskal-Oberman (KO) kinetic contribution from thermal particles in the Porcelli model. In the following estimates, the KOcontribution is replaced by the drift kinetic energy perturbations associated with trapped thermal particles as shown in Fig. 6(b). The latter is supposed to be more accurate than the KO-value, since no high-frequency approximation is assumed. The quantity $\delta \hat{W}$ from Eqs. (15)-(16) is the normalized total perturbed potential energy, including the core contribution and the kinetic contribution from alpha particles. In other words, we have

$$
\begin{gathered}
\delta \hat{W}_{\text {core }}=\delta \hat{W}_{\text {mhd }}+\delta \hat{W}_{\text {th }} \\
\delta \hat{W}=\delta \hat{W}_{\text {core }}+\delta \hat{W}_{\alpha}
\end{gathered}
$$

where $\delta \hat{W}_{t h}$ denotes the thermal contributions $\left(t_{P}+t_{B}\right)$ replacing the KO-contribution in the Porcelli 
sawtooth model. $\delta \hat{W}_{\alpha}$ is the perturbed energy due to precessional drifts of trapped alpha particles $\left(\alpha_{p}\right)$. Note that, following the convention in the Porcell model, all the above perturbed potential energy components are normalized by the normalized factor $\operatorname{Re}[\delta I] \cdot\left(0.072 s_{1}+0.077\right)$.

Before proceeding with the analysis of sawtooth-triggering conditions (14)-(16), we point out that the Porcelli model neglects the imaginary part of the potential energy $\operatorname{Im}[\delta W]$, which is roughly valid for the internal kink mode (sawteeth). In term of the real part of the potential energy $\operatorname{Re}[\delta W]$, kinetic contribution from either thermal or alpha particles provides a stabilizing effect for the EU DEMO plasma, as shown by Fig. 6(b). Furthermore, the absolute value of the MHD energy, $\delta \hat{W}_{\text {mhd }}$, is larger than the kinetic energy from thermal particles (marked with " $t$ t tB"), resulting in $\delta \hat{W}_{\text {core }}$ being negative according Eq. (18). This means that the internal kink remains unstable with thermal kinetic effects. On the other hand, $-\delta \hat{W}_{\text {core }}=3.84 \times 10^{-3}$ is less than $c_{h} \omega_{D \alpha} \tau_{A}=8.74 \times 10^{-3}$, indicating full stabilization of the mode by alpha particles. In fact, the total perturbed energy $\delta \hat{W}=9.85 \times 10^{-3}$ is indeed positive. It is also evident that condition (15) is not satisfied according to Tab. 1. This leaves us with condition (16). Part of the condition, $\omega_{*}^{\text {thi }}<c_{*} \gamma_{\rho}$, is easily satisfied for this EU-DEMO plasma, but the other part, $\delta \hat{W}<\hat{\rho}$, is not satisfied. Violation of all three Porcelli criteria (14)-(16) indicates that sawtooth crash will not be triggered in the EU DEMO reference plasma, without local modification of the magnetic shear (i.e. with $s 1 \approx 0.6$ ).

We should point out that, although the Porcelli model predicts strong stabilization of the internal kink mode and thus no sawtooth crash for the reference EU DEMO plasma, the MARS-K eigenvalue computations still show a residual instability (Fig. 6(a)), albeit at much reduced mode growth rate by the drift kinetic stabilization. The difference in the results between two models can be attributed to couple of factors. (i) The MARS-K eigenvalue computations follow a nonperturbative MHD-kinetic hybrid approach, whilst Porcelli model is based on the perturbative energy analysis. The main difference is that the non-perturbative approach allows self-consistent modification of mode eigenfunction by drift kinetic effects. This normally leads to a more unstable mode (essentially because we give the mode more freedom to develop itself). (ii) The Porcelli model includes finite Larmor radius (FLR) effect (in both criteria (15) and (16)) whilst this physics is absent in MARS-K with standard MHD formulation. This FLR effect is typically strongly stabilizing for the internal kink mode. (iii) The Porcelli model ignores $\operatorname{Im}[\delta W]$, which also affects the mode stability. In this regard, we mention a previous internal kink study for ITER [8], that included both $\operatorname{Im}[\delta W]$ and the inertial enhancement effect due to bulk thermal ion kinetic effects, while still following the perturbative approach.

As a final remark, we mention the possibility of the fishbone drive in this EU DEMO plasma. As it is well known, fishbone oscillations can be triggered by the precessional drift resonance of energetic particles, when the following condition is met [40] (assuming that the on-axis safety factor is below or close to 1)

$$
\beta_{p \alpha}^{*}>\beta_{p \alpha}^{f i s h}=0.45 s_{1} \omega_{D \alpha} \varepsilon_{1}^{-3 / 2}
$$

where $\beta_{p \alpha}^{*}$ is poloidal beta of alpha particles, which depends on the pressure gradient of alphas within the $q=1$ region. $\varepsilon_{1}=r_{1} / R$ is the inverse aspect ratio of the $q=1$ surface. The threshold value, $\beta_{p \alpha}^{f i s h}$, for fishbone triggering, is smaller without regard to the thermal ion Landau damping. We 
have estimated that $\beta_{p \alpha}^{*}$ and $\beta_{p \alpha}^{f i s h}$ robustly have the same order of magnitude, and the value of $\beta_{p \alpha}^{*}$ is slightly greater than $\beta_{p \alpha}^{\text {fish }}$ for the EU DEMO plasma under consideration (with $s_{1} \approx 0.6$ ). This suggests that the fishbone may be driven by alpha particles in EU DEMO. On the other hand, it is the common wisdom that fishbones will not notably change the sawtooth activity (due to large separation of the modes frequency) [7].

\subsubsection{Effect of local magnetic shear}

Now, we turn to investigating the possibility of sawtooth triggering, via modification of the local magnetic shear near the $q=1$ surface for the EU DEMO plasma. In the following, we again consider the drift kinetic contributions from both thermal and fusion born $\alpha$ particles. According the Porcelli sawtooth crash criteria, there are two ways to cause sawtooth crash with increasing the local magnetic shear $s_{1}$. (i) Increasing $s_{1}$ makes it easier to satisfy the condition $\omega_{*}^{t h i}<c_{*} \gamma_{\rho}$ in the ionkinetic regime, since $\gamma_{\rho}$ is proportional to $s$ in this case. (ii) Since the drift kinetic contribution to the perturbed potential energy is stabilizing (i.e. positive) and is inversely proportional to $s_{1}$, it is possible to decrease this stabilizing contribution by increasing the local magnetic shear and consequently to further destabilize the internal kink. (Note that this is achievable despite the fact that the destabilization role of the fluid potential energy is reduced by increasing $s_{1}$.)

In what follows, we choose three $s 1$ values that are larger than that of the reference plasma without local current drive, i.e., $s_{1}=1.5, s_{1}=2.5$ and $s_{1}=3.5$. The corresponding MARS-K results are reported in Figs. 7 and 8. Figure 7(a) shows that the real part of $-\delta \widehat{W}_{\text {core }}=-\delta \widehat{W}_{\text {mhd }}-$ $\delta \widehat{W}_{t h}$ increases with increasing $s_{1}$ (red curve). As stated above, $\operatorname{Re}\left[-\delta \widehat{W}_{m h d}\right]$ decreases with increasing $s 1$, indicating that the kinetic contribution from thermal particles $\left(\operatorname{Re}\left[\delta \widehat{W}_{t h}\right]\right)$ is positive and weakens with increasing $s_{1}$. Similarly, it can be deduced from Fig. 7 (b) (red curve) that the kinetic stabilization from alpha particles $\left(\operatorname{Re}\left[\delta \widehat{W}_{\alpha}\right]\right)$ also gradually declines with increasing $s$. In general, we find that the drift kinetic stabilization weakens with increasing the local magnetic shear near the $q=1$ surface.

Now considering the sawtooth crash criterion (14), Fig. 7(a) shows that $-\delta \hat{W}_{\text {core }}$, though substantially increases with $s$, is still less than $c_{h} \omega_{D \alpha} \tau_{A}$ even at $s_{1}=3.5$. This means that the internal kink is still fully stabilized by alphas at increased local magnetic shear, according to the Porcelli criterion (14) (and assuming $c_{\mathrm{h}}=0.4$ ). On the other hand, the MARS-K computed total potential energy $\operatorname{Re}[\delta \hat{W}]$ changes from positive to negative values as $s_{1}$ increases (Fig. 7(b)), making the condition (15), i.e. $-\operatorname{Re}[\delta \hat{W}]>0.5 \omega_{*}^{\text {thi }} \tau_{A}$, satisfied at $s_{1}=3.5$. This shows that sawtooth crash is indeed possible at increased local magnetic shear. At first glance, this conclusion appears to be contradicting that obtained with condition (14). The reason is in the choice of the numerical factor $c h$, which is sensitive to the plasma equilibrium. The value of $c_{h}$ in EU DEMO is probably smaller than that estimated for ITER. Figure 7(b) also shows that the Porcelli criterion (16), for the onset of internal kink in the ion-kinetic regime, can be satisfied in a range of (increasing) local magnetic shear. This is because the condition $\omega_{*}^{\text {thi }}<c_{*} \gamma_{\rho}$ is always satisfied, hence the threshold condition is $\delta \hat{W}=\hat{\rho}$, which holds at $s_{1} ; 2.8$. In summary, Fig. 7 shows that sawtooth crashes can occur in EU DEMO at enhanced local magnetic shear near the $q=1$ surface, by satisfying 
at least one of the Porcelli criteria.

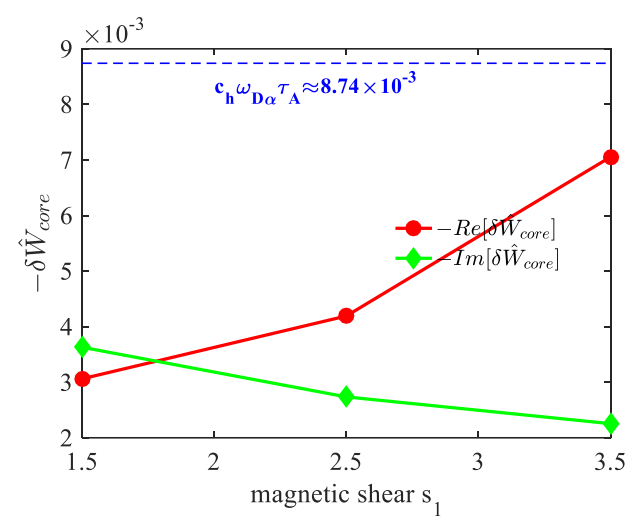

(a)

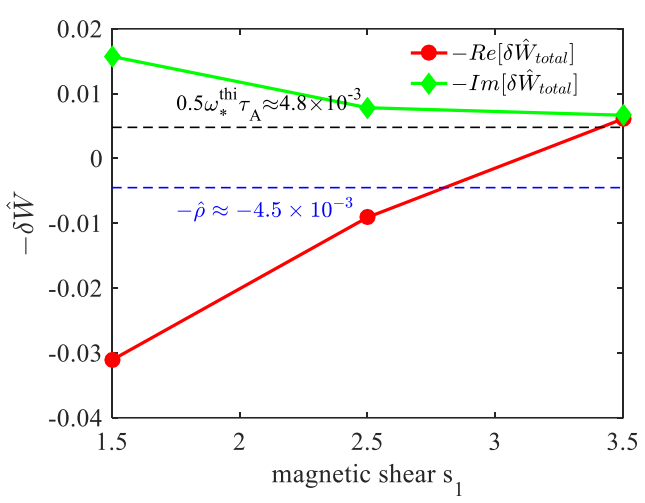

(b)

Figure 7. Comparison of the MARS-K computed perturbed potential energies with various Porcelli criteria for the sawtooth onset, for (a) the normalized potential energy $-\delta \hat{W}_{\text {core }}$ including drift kinetic contributions from thermal particles, and (b) the normalized potential energy $-\delta \hat{W}$ including kinetic contributions from both thermal and fusion born alpha particles. Varied is the local equilibrium magnetic shear $s_{1}$ at the $q=1$ surface. The Porcelli criterion $c_{h} \omega_{D h} \tau_{A}$ is associated with trapped alpha stabilization due to fast precessional drift. The criterion is associated with diamagnetic stabilization, and $\hat{\rho}=\rho_{i} / r_{1}=4.5 \times 10^{-3}$ is the normalized thermal ion Larmor radius. Other parameters are $\eta=1 \times 10^{9}, b / a=1$.

Meanwhile, the MARS-K non-perturbative MHD-kinetic hybrid eigenvalue computations show more robust occurrence of sawtooth crash (i.e. the presence of unstable internal kink), but in the absence of the diamagnetic stabilization. Figure 8 compares the computed eigenvalue and perturbed potential energy of the internal kink, for equilibria with $s_{1}=1.5,2.5$ and 3.5. The mode remains unstable with large growth rates, at all three values of $s_{1}$ and with inclusion of various drift kinetic effects (Fig. 8(a)). The latter provide a stabilization effects to the mode, similar to the Porcelli model. Porcelli model based on the perturbative approach. On the other hand, drift kinetic contributions, in particular that of fusion born alphas, also provide large imaginary part of the energy perturbation that is comparable to the real part by magnitude (Fig. 8(b)). The imaginary part of the drift kinetic energy perturbation, being not included into the Porcelli model, also plays a role in the mode stability. 


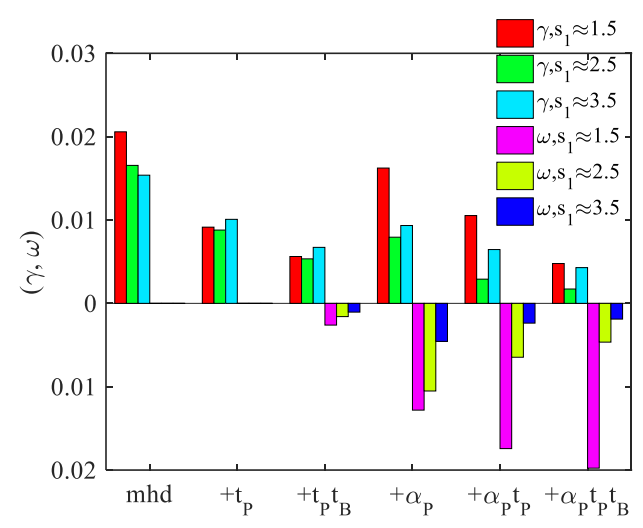

(a)

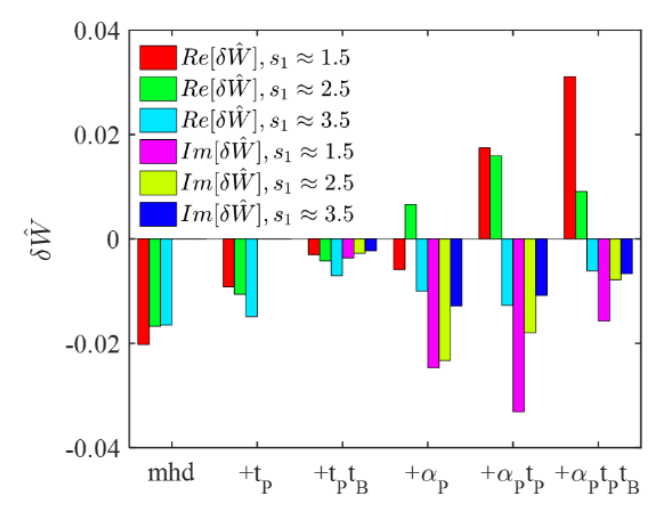

(b)

Figure 8. (a) The MARS-K computed growth rate $\gamma$ and mode frequency $\omega$, normalized by the onaxis Alfven frequency, for the $n=1$ internal kink, with each group representing inclusion of a specific drift kinetic effect. Within each group, comparison is also made among different choices of local magnetic shear $s_{1}$. (b) The real and imaginary parts of the perturbed potential energies due to MHD as well as various drift kinetic contributions - the precession drift of trapped thermal particles $\left(t_{\mathrm{P}}\right)$ or fusion born alpha particles $\left(\alpha_{\mathrm{P}}\right)$, the bounce motion of trapped thermal ions $\left(t_{\mathrm{B}}\right)$ - within each group. Compared are also different groups with varying local magnetic shear at the $q=1$ surface: $s_{1}=1.5, s_{1}=2.5, s_{1}=3.5$. Other parameters are $\eta=10^{9}, b / a=1$.

Our study so far did not include the influence of plasma equilibrium flow on the internal kink instability. It is well known that the (uniform) toroidal flow can provide a stabilizing effect on the internal kink, via the so-called gyroscopic mechanism. For instance, It has been demonstrated that a sonic-range toroidal plasma rotation provides strong stabilization to the mode [41]. Furthermore, sonic-range toroidal flow can significantly modify the plasma equilibrium due to centrifugal effect, which in turn leads to important consequences in the flow stabilization/destabilization of the internal kink [42]. On the other hand, slow plasma flow is likely expected for the EU DEMO plasmas (similar to ITER). Assuming an ITER-like sheared toroidal flow profile [40], with flow amplitude well within the sub-sonic level, we carried out the MARS-F stability computations for the EU DEMO plasma, and found little impact of the plasma flow on the internal kink instability. This also agrees with findings from an early study with similar assumptions [17].

\section{Summary}

For a reference EU DEMO plasma, we carried out systematic investigation on the internal kink instability and sawtooth crash triggering, with particular emphasis on the role of local modification of the magnetic shear near the $q=1$ surface via local current drive. Employed are both fluid (MARSF) and MHD-kinetic hybrid (MARS-K) models that take into account full toroidal effects, as well as the semi-analytical Porcelli model for sawtooth crash prediction. The rational for considering both MARS-K and Porcelli models is to take advantages of both approaches (e.g. non-perturbative kinetic closure, full toroidal geometry with MARS-K, FLR and ion-kinetic layer physics in the 
Porcelli model).

The presence of a finite edge current in the original equilibrium, designed for EU DEMO, leads to a peeling mode instability with the dominant $m / n=5 / 1$ harmonic (in the absence of a closing fitting ideal wall). In order to eliminate the interference of this instability, we slightly modified the toroidal current density profile near the plasma edge in our modeling, by making the current density vanish at the plasma boundary. This modification has little impact on the $m / n=1 / 1$ internal kink instability that we are interested in.

According to the Porcelli model, the internal kink is stable in the target EU DEMO plasma, due to fusion born alpha stabilization as well as the diamagnetic stabilization. However, sawtooth crash can be triggered by additional local current drive near the $q=1$ surface, which enhances the local magnetic shear. In particular, sawtooth crashes can occur by accessing the (unstable) ionkinetic regime, at $s_{1} \sim 2.8$ or higher in EU DEMO, based on the Porcelli criterion. The local magnetic shear $s_{1}$ stabilizes the internal kink mode based on the fluid model, but destabilizes the mode when the drift kinetic effects are included as predicted by the Porcelli model.

Similar trend is obtained by direct, non-perturbative MARS-K MHD-kinetic hybrid eigenvalue computations. On the other hand, MARS-K predicts less stabilization of the internal kink mode and hence a more robust access to the sawtooth regime, than that by the Porcelli model. This discrepancy can be largely attributed to the following three factors. (i) The MARS-K eigenvalue model is a nonperturbative model, whilst the Porcelli model is a perturbative model. (ii) The Porcelli model includes finite Larmor radius (FLR) effect, whilst this physics is absent in the standard single fluid MHD formulation within MARS-K. (iii) The Porcelli model ignores the imaginary part of the energy perturbations.

With the expectation of slow plasma flow in EU DEMO, a static equilibrium is assumed in most of the present study. A sub-sonic equilibrium toroidal flow is found to only weakly affect the mode stability by MARS-F. The effect of energetic particles from auxiliary heating is also neglected in this work. This contribution may provide additional stabilization to the internal kink mode [43], although the dominant effect may still come the fusion born $\alpha$-particles in EU DEMO due to large pressure fraction. More quantitative investigation on this issue remains a future work.

\section{Acknowledgments}

LNZ thanks Drs Z R Wang, G Z Hao, F Wang, C Liu, G L Xia, Y L He, J Ren, S Wang, X Yang, S $\mathrm{Y}$ Yang and Y Wang for useful discussions and help. This work is supported by "the Fundamental Research Funds for the Central Universities" (grant number 3132020181), and by National Natural Science Foundation of China (NSFC) (Grant Nos. 11975062 and 11605021). 
[1] von Goeler S, Stodiek W, Sauthoff N 1974 Phys. Rev. Lett. 33 1201-1203

[2] Nave M F F et al 2003 Nucl. Fusion 431204

[3] Campbell D J et al 1988 Phys Rev Lett 60 2148-2151

[4] Nave M F F, Gorelenkov N N, McClements K G, Allfrey S J, Balet B, Borba D N, Lomas P J, Manickam J, Jones T T C, Thomas P R 2002 Nucl. Fusion 42281

[5] Sauter O et a/ 2002 Phys Rev Lett 88105001

[6] Gude A, Gunter S, Maraschek M, Zohm H, Team t A U 2002 Nucl. Fusion 42833

[7] Porcelli F, Boucher D, Rosenbluth M N 1996 Plasma Phys. Control. Fusion 38 2163-2186

[8] Hu B, Betti R, Manickam J 2006 Phys. Plasmas 13112505

[9] Chapman I T et al 2007 Plasma Phys. Control. Fusion 49 B385-B394

[10] Chapman I T 2011 Plasma Phys. Control. Fusion 53013001

[11] Pinches S D, Chapman I T, Lauber P W, Oliver H J C, Sharapov S E, Shinohara K, Tani K 2015 Phys. Plasmas 22021807

[12] Romanelli F, Barabaschi P, Borba D, Federici G, Horton L, R. Neu, D. Stork, H. Zohm, 2012.

[13] Federici $G$ et al 2014 Fusion Eng. Design 89 882-889

[14] Somjinda B, Wisitsorasak A, Onjun T 2020 Nucl. Fusion 60066013

[15] Wu T, He H, Liu Y, Liu Y, Hao G Z, Zhu J 2018 Phys. Plasmas 25052504

[16] Bondeson A, Bussac M N 1992 Nucl. Fusion 32513

[17] Wu T, Liu Y, Liu Y, Zhou L, He H 2019 Phys. Plasmas 26102102

[18] White R B, Rutherford P H, Colestock P, Bussac M N 1988 Phys Rev Lett 60 2038-2041

[19] Coppi B, Migliuolo S, Pegoraro F, Porcelli F 1990 Physics of Fluids B: Plasma Physics 2927 943

[20] Eriksson L G et al 2004 Phys Rev Lett 92235004

[21] Lennholm M et al 2009 Phys Rev Lett 102115004

[22] Bhatnagar V P, Start D F H, Jacquinot J, Chaland F, Cherubini A, Porcelli F 1994 Nucl. Fusion 341579

[23] Westerhof E et al 2002 Nucl. Fusion 421324

[24] Mantsinen M et a/ 2002 Plasma Phys. Control. Fusion 441521

[25] Eriksson L G et al 2006 Nucl. Fusion 46 S951-S964

[26] Liu Y, Chu M S, Chapman I T, Hender T C 2008 Phys. Plasmas 15112503

[27] Liu Y Q, Bondeson A, Fransson C M, Lennartson B, Breitholtz C 2000 Phys. Plasmas 7 3681-3690

[28] Berkery J W, Liu Y Q, Wang Z R, Sabbagh S A, Logan N C, Park J-K, Manickam J, Betti R 2014 Phys. Plasmas 21052505

[29] Wang Z, Park J-K, Liu Y, Logan N, Kim K, Menard J E 2014 Phys. Plasmas 21042502

[30] Liu Y, Chu M S, Chapman I T, Hender T C 2009 Nucl. Fusion 49035004

[31] Liu Y 2010 Nucl. Fusion 50095008

[32] Liu Y et al 2010 Plasma Phys. Control. Fusion 52104002

[33] Menard J E, Wang Z, Liu Y, Bell R E, Kaye S M, Park J K, Tritz K 2014 Phys. Rev. Lett. 113

[34] Wang Z R, Lanctot M J, Liu Y Q, Park J K, Menard J E 2015 Phys. Rev. Lett. 114145005

[35] Siccinio M et a/ 2020 Fusion Eng. Design 156111603

[36] Garavaglia S et a/2020 Fusion Eng. Design 156111594

[37] Lütjens H, Bondeson A, Sauter O 1996 Computer Physics Communications 97219

[38] Guo S C, Xu X Y, Liu Y Q, Wang Z R 2016 Nucl. Fusion 56056006 
[39] Hao G Z, Yang S X, Liu Y Q, Wang Z X, Wang A K, He H D 2016 Phys. Plasmas 23062105

[40] Chen L, White R B, Rosenbluth M N 1984 Phys. Rev. Lett. 52 1122-1125

[41] C. Wahlberg a A B 2000 Phys. Plasmas 7923

[42] Wahlberg C, Chapman I T, Graves J P 2009 Phys. Plasmas 16112512

[43] Graves J P 2004 Phys Rev Lett 92185003 